\title{
Genetic Algorithm Search for Stent Design Improvements
}

\author{
K. Tesch, M.A. Atherton \& M.W. Collins, \\ South Bank University, London, UK
}

\begin{abstract}
This paper presents an optimisation process for finding improved stent design using Genetic Algorithms. An optimisation criterion based on dissipated power is used which fits with the accepted principle that arterial flows follow a minimum energy loss. The GA shows good convergence and the solution found exhibits improved performance over proprietary designs used for comparison purposes.
\end{abstract}

\section{Introduction}

Stents are metallic cage like structures (Figure 1) that are inserted into an artery blocked by calcified plaque (stenosis). Stents differ significantly in shape, cross-sections, and other details, which affect the haemodynamics of the blood flow through the treated region.

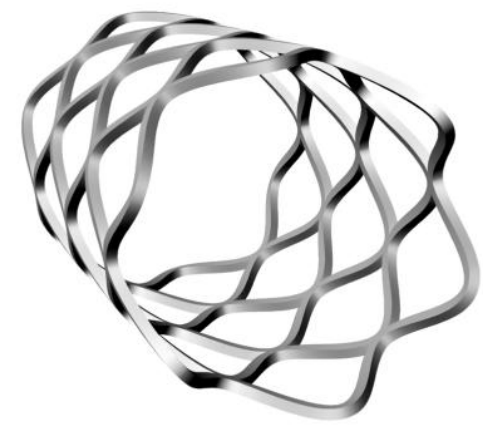

Fig. 1. Palmaz-Schatz stent

Early designs were woven from round-section wire but current designs are laser-cut cylinders enabling a wider range of designs. In its unexpanded form a stent is delivered on an angioplasty balloon and receives its final shape after expansion beyond its elastic limit.

\section{Stent Pattern Description}

Stent design is deceptively simple but the degrees of freedom of the patterns mean there are thousands of potential solutions. Also these shapes can be rather complicated and therefore it is difficult to describe all the shapes using simple descriptions. In this study the stent patterns were represented by five main features as described below.

From Figure 1 the stent is seen to comprised of strut arranged to make the patterns. Three values of strut thickness were used ranging form 0.08 to $0.10 \mathrm{~mm}$ (Figure 2).

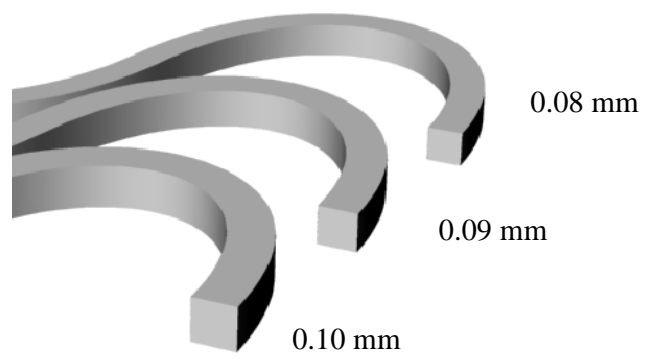

Fig. 2. Strut Thickness, d 
The shape of the strut cross-section was represented as a ratio of thickness to width (Figure 3) based upon the actual thickness value used from Figure 2.

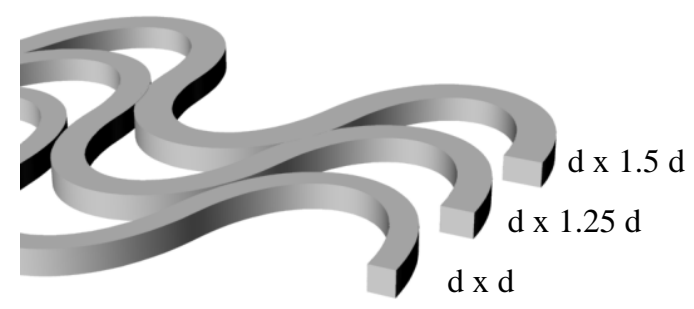

Fig. 3. Strut Thickness: Width ratio

Generally the stent patterns are symmetrical therefore a skew parameter was introduced in order to explore asymmetry. A skew value of 0.5 represents symmetrical pattern and value of 0.9 produces asymmetry through bringing the two peaks of the pattern closer together (Figure 4).

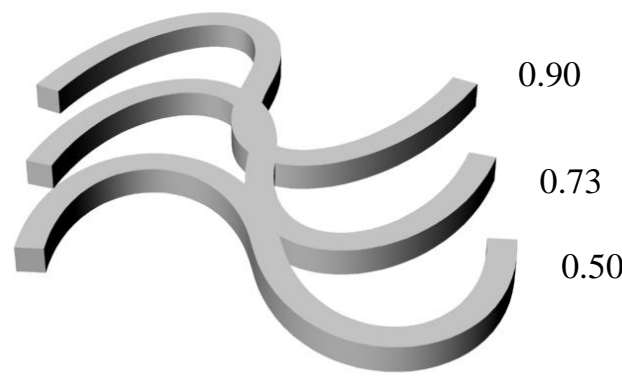

Fig. 4. Pattern Skew

Repeating Pattern determines whether the next stents segment is merely a copy or it is a mirror image of the existing segment. If a segment is copied then an artificial link must be added to join the two segments together. A mirror operation does not need any linking elements because segments are joined naturally (Figure 5).
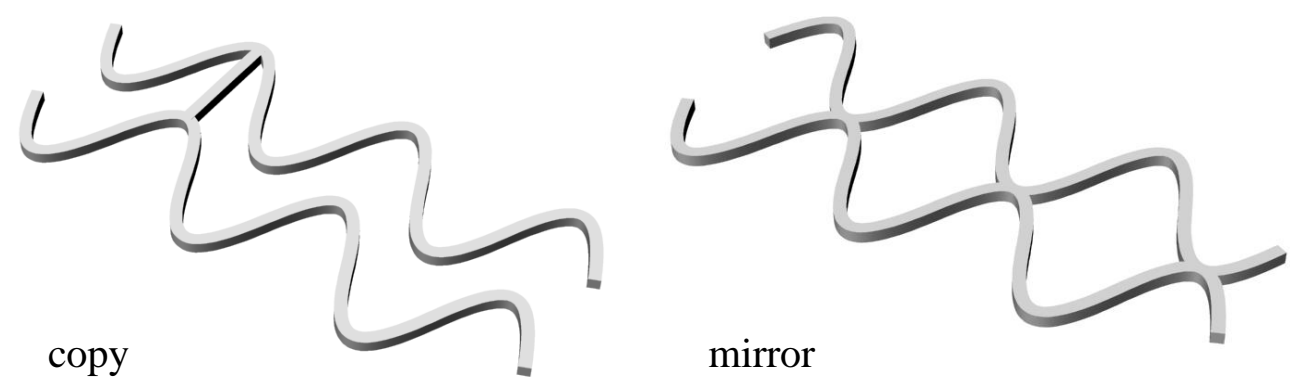

Fig. 5. Repeating Pattern

Shape Order (Figure 6) defines the degree of curvature of the segments. In this particular case ' $1{ }^{\text {st }}$ order' produces a sharp definition and ' 2 nd order' produces a smoother definition.
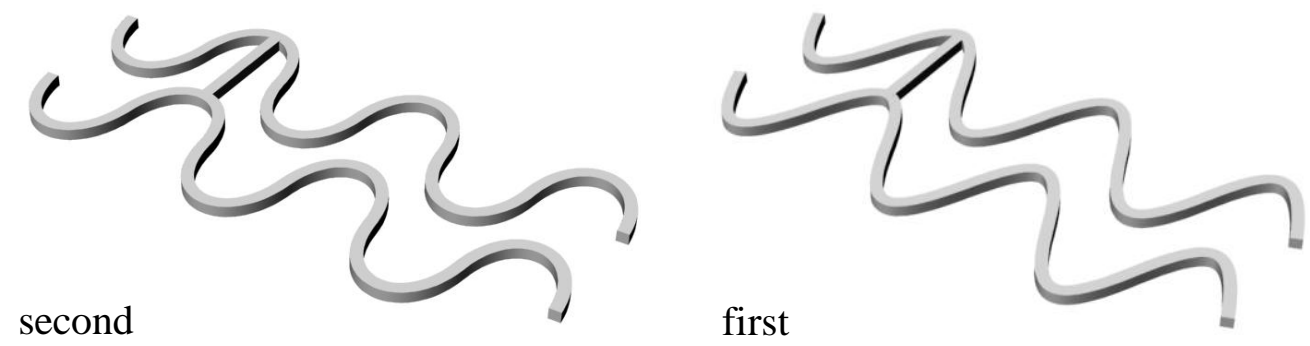

Fig. 6. Shape Order 
The five variables described above allow to coverage of a relatively large design space. However it is only a simplification and one could imagine a more complex description.

Table 1. Stent pattern description

\begin{tabular}{|l|l|}
\hline Name & Range \\
\hline Strut Thickness d (mm) & $0.08-0.10$ \\
\hline Strut Thickness:Width ratio & $1: 1-1: 1.5$ \\
\hline Pattern Skew & $0.5-0.9$ \\
\hline Repeating Pattern & Copy/Mirror \\
\hline Shape Order & $1^{\text {st }}$ and $2^{\text {nd }}$ \\
\hline
\end{tabular}

As a first approximation, the design was parameterised according to Table 1 in order to simplify the search. For computational purposes, it is convenient to work with a partial model of a stent rather than a full $3 \mathrm{D}$ model

\section{Physics}

Blood flow behaviour may be described by Navier-Stokes and continuity equations (Equations 1).

$$
\left.\begin{array}{c}
\frac{\mathrm{d} \rho}{\mathrm{dt}}+\rho \nabla \cdot \overrightarrow{\mathrm{U}}=0 \\
\rho \frac{\mathrm{d} \overrightarrow{\mathrm{U}}}{\mathrm{dt}}=\rho \overrightarrow{\mathrm{g}}+\nabla \cdot \boldsymbol{\sigma}
\end{array}\right\}
$$

The general form has too many unknown variables. We need another equation known as Newtonian hypothesis. It describes a linear relation between stress tensor $\boldsymbol{\sigma}$ and strain rate tensor $\mathbf{D}$

$$
\boldsymbol{\sigma}=-\left(\mathrm{p}+\frac{2}{3} \mu \nabla \cdot \overrightarrow{\mathrm{U}}\right) \boldsymbol{\delta}+2 \mu \mathbf{D}
$$

where strain rate tensor is given by

$$
\mathbf{D}:=\frac{1}{2}\left(\nabla \overrightarrow{\mathrm{U}}+(\nabla \overrightarrow{\mathrm{U}})^{\mathrm{T}}\right)
$$

Having 10 scalar formulas ( 1 scalar +1 vector +1 symmetrical tensor) we still have 12 unknown variables: $\rho, \mathrm{U}_{\mathrm{x}}, \mathrm{U}_{\mathrm{y}}, \mathrm{U}_{\mathrm{z}}, \sigma_{\mathrm{xx}}, \sigma_{\mathrm{yy}}, \sigma_{\mathrm{zz}}, \tau_{\mathrm{xy}}, \tau_{\mathrm{xz}}, \tau_{\mathrm{yz}}, \mathrm{p}, \mu$. We may treat blood as an incompressible medium then

$$
\rho=\text { const } \mu=\text { var }
$$

The 12 scalar formulas can be rewritten now as follows

$$
\left.\begin{array}{l}
\nabla \cdot \overrightarrow{\mathrm{U}}=0 \\
\rho \frac{\mathrm{d} \overrightarrow{\mathrm{U}}}{\mathrm{dt}}=-\nabla \mathrm{p}+\mu \nabla^{2} \overrightarrow{\mathrm{U}}+2 \mathbf{D} \cdot \nabla \mu
\end{array}\right\}
$$

There are 4 equations and 5 unknown variables: $\mathrm{U}_{\mathrm{x}}, \mathrm{U}_{\mathrm{y}}, \mathrm{U}_{\mathrm{z}}, \mathrm{p}, \mu$. It is necessary to decide whether to treat blood as Newtonian or non-Newtonian. A non-Newtonian behaviour allows a relationship between viscosity and velocity gradients according to the following power law

$$
\mu=\mathrm{k} \cdot \gamma^{\mathrm{n}-1}
$$

where $\mathrm{k}$ and $\mathrm{n}$ are constants.

e.g. blood

and shear strain rate $\gamma$ for a general 3D case

$$
\mathrm{n}=0.61, \mathrm{k}=0.042\left[\mathrm{~kg} \mathrm{~m}^{-1} \mathrm{~s}^{-1.39}\right]
$$

$$
\gamma=\sqrt{2 \mathbf{D}: \mathbf{D}}
$$

Finally, the fifth non-linear equation is established, which describes the non-Newtonian behaviour of blood 


$$
\mu=\mathrm{k}(2 \mathbf{D}: \mathbf{D})^{\frac{\mathrm{n}-1}{2}}
$$

Formulas (5) and (8) may be solved numerically using Finite Volume Method or Finite Elements Method. However for either approach the stent geometry first must be created and then discretised (Figure 7).

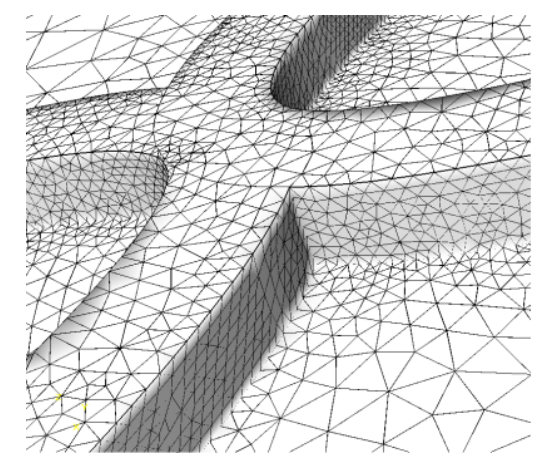

Fig. 7. CFD Mesh example

The descretisation process and mesh quality are crucial to the accuracy of results. Therefore special care must be taken near the stent surfaces where velocity gradients are relatively high. Different types of element were implemented in order to improve mesh quality and the convergence.

\section{Objective Fitness Function}

One could imagine a lot of different fitness functions. It is believed that Wall Shear Stress plays the most important role in bio-medical flows [2]. However as WSS is distributed along the surface it cannot be directly used as a performance measure in driving a search algorithm that needs a number(s). Indeed two different WSS distributions cannot be compared directly, yet it is possible to define such a performance based on that distribution. It should be borne in mind that if a number is generated from the 3D distribution then information is always lost.

Dissipated power is introduced here as an alternative performance measure. Let us consider a form of the Gibbs equation that has the shape of an energy equation:

$$
\rho \frac{\mathrm{de}}{\mathrm{dt}}=\mathrm{Ts}_{\mathrm{m}}^{+}+\frac{\mathrm{p}}{\rho} \frac{\mathrm{d} \rho}{\mathrm{dt}}+\lambda \nabla^{2} \mathrm{~T}
$$

where $\mathrm{T}$ is temperature and $\mathrm{Ts}_{\mathrm{m}}^{+}$is the intensity of entropy production. As blood is incompressible and we neglect the heat conductivity we have

$$
\rho \frac{\mathrm{de}}{\mathrm{dt}}=\mathrm{Ts}_{\mathrm{m}}^{+}
$$

In other words the dissipated energy causes an incremental change of internal energy. The effect of this energy dissipation is an increase of local temperature. Intensity of entropy production may be calculated from the velocity field (for an incompressible medium).

$$
\mathrm{Ts}_{\mathrm{m}}^{+}=\boldsymbol{\sigma}: \nabla \overrightarrow{\mathrm{U}}
$$

It can be proved that not all of the work in Equation 12 is converted into kinetic energy

$$
\mathrm{dL}_{\mathrm{V}}+\mathrm{dL}_{\mathrm{S}}=\mathrm{dE}_{\mathrm{kin}}+\mathrm{dt} \iiint_{\mathrm{V}} \mathrm{Ts}_{\mathrm{m}}^{+} \mathrm{dxdydz}
$$

The energy that is not converted, is dissipated. Therefore dissipated power is defined as follows

$$
\mathrm{N}_{\mathrm{diss}}:=\iiint_{\mathrm{V}} \mathrm{Ts}_{\mathrm{m}}^{+} \mathrm{dxdydz}
$$

or dissipated energy

$$
\mathrm{E}_{\mathrm{diss}}:=\int_{\mathrm{t}} \mathrm{dt} \iiint_{\mathrm{V}} \mathrm{Ts}_{\mathrm{m}}^{+} \mathrm{dxdydz}
$$

Minimising such an objective fitness function (Equation 13) helps us to search for a stent shape with the smallest possible energy losses. 


\section{Parameter Encoding}

Two alleles were used for both 'ratio' and 'thickness' in encoding the GA chromosome. As these variables have only three values and the alleles can represents four values $\left(2^{2}\right)$ then a dummy level was necessary. Therefore the fourth value in the allele combination was made equal to the third value.

Table 2.

\begin{tabular}{|l|l|l|l|l|}
\hline Name & Range & Step & Type & Bits \\
\hline Strut Thickness d (mm) & $0.08-0.11 \mathrm{~mm}$ & 0.01 & Floating & 2 \\
\hline Strut Thickness:Width ratio & $1-1.75$ & 0.25 & Floating & 2 \\
\hline Pattern Skew & $0.5-0.9$ & 0.057143 & Floating & 3 \\
\hline Repeating Pattern & $0-1$ & - & Boolean & 1 \\
\hline Shape Order & $1-2$ & 1 & Integer & 1 \\
\hline
\end{tabular}

The full parameter encoding for the stent chromosome is shown below. The total length of chromosome is 9 , which represents 5 variables.

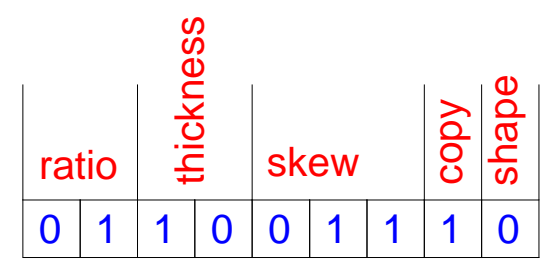

Fig. 8. Parameter encoding

The above encoding presents us with $2^{9}=512$ possibilities. It is necessary to mention that not all of them are unique because of the dummy level, which means there are 288 unique cases.

\section{Optimisation Values and Results}

Due to the relatively short chromosome length and the high cost of calculation, the population size was chosen to be 10 . Crossover probability was set at 0.75 and mutation probability at 0.02 to avoid local extreme convergence.

The total number of generations passed to convergence was 9. During the calculation process 15 mutations and 38 crossovers were produced. The total number of unique stent shapes tested was 28 , which is about $10 \%$ of the whole design space.

The tournament method of selecting individuals for crossover was used for two reasons. Firstly the roulette method was unsuitable for dealing with a minimised objective function and also it needed function scaling to avoid random wandering. Secondly our experience suggested it as the superior approach. A tournament size of 2 was used.

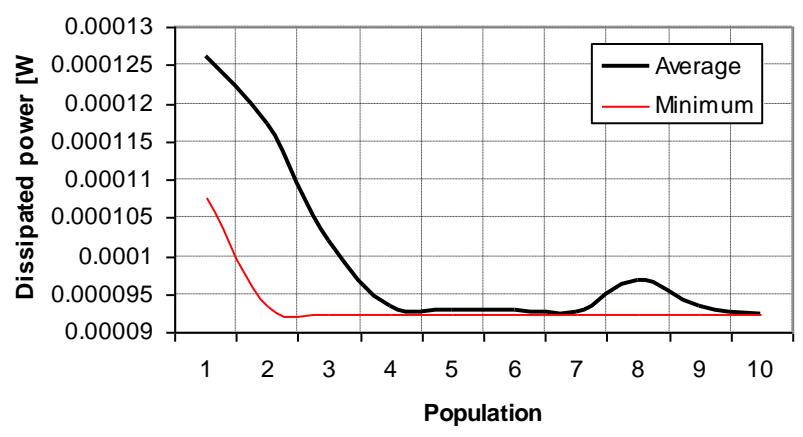

Fig. 9. Convergence 
The optimal solution obtained from GA optimisation process is shown below.

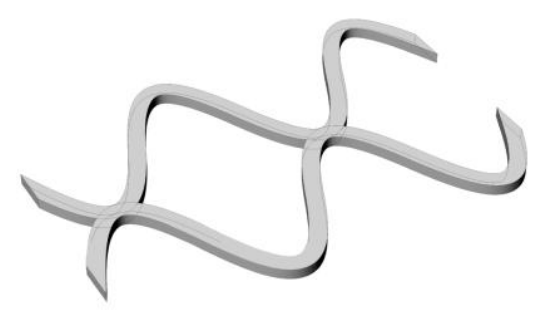

Fig. 10. The GA optimal solution

The set of design variables defining the 'optimal solution' is shown in Table 3.

Table 3.

\begin{tabular}{|l|l|}
\hline Name & Value \\
\hline Strut Thickness d $(\mathrm{mm})$ & 0.08 \\
\hline Strut Thickness:Width ratio & $1: 1.5$ \\
\hline Pattern Skew & 0.5 \\
\hline Repeating Pattern & Mirror \\
\hline Shape Order & $1^{\text {st }}$ \\
\hline
\end{tabular}

\section{Discussion \& Conclusions}

The GA is limited in terms of its inability to interpolate the discrete values of design variables explored. However, the big advantage of the GA method was seen to be in its quick convergence. One could ask if the GA found the true optimum, which of course cannot be answered without knowing all the possible solutions. Indeed the GA solution compared favourably with that from another solution.

It is clean that optimal shape depends on the objective fitness function. Dissipated power prefers shape that looks similar to a Palmaz-Schatz design. Yet other performance measure would presumably give different results. To answer that question it is necessary to introduce a way of classifying shape performance based on WSS. This will be investigated in our future work.

\section{References}

[1] Goldberg, D.E., "Genetic Algorithms in Search Optimization and Machine Learning”, Addison Wesley, (1989).

[2] Sigwart, U., “Coronary stents: will they survive?”, pp. 169-177 in 'Coronary Stents' (Eds. U. Sigwart and G. I. Frank). Springer-Verlag (1992).

[3] Haupt R.L., Haupt S.E. "Practical Genetic Algorithms” John Wiley \& Sons, Inc. (1998) 